\title{
SPECT-MPI in evaluation of liver transplant candidates: Is the evidence mounting?
}

\author{
Javier Gomez, MD, MSc, FACC, FASNC ${ }^{\mathrm{a}}$ \\ a Division of Cardiology, John H. Stroger Jr, Hospital of Cook County, Chicago, IL
}

Received Jun 9, 2020; accepted Jun 9, 2020

doi: $10.1007 / \mathrm{s} 12350-020-02240-y$

\section{See related article, pp. 2876-2891}

According to recent data from the United Network for Organ Sharing (UNOS), there are 12,719 patients with end stage liver disease (ESLD) awaiting orthotopic liver transplant (OLT) in the US. ${ }^{1}$ The number of ESLD patients who await liver transplant has been rising in recent years. Given the limited number of donors, optimal selection of candidates with acceptable cardiovascular risk is a crucial step to ensure the most favorable results. The ideal screening method to predict cardiovascular outcomes in patients who are considered for OLT continues to be a subject of debate. ${ }^{2}$ Thus far, data evaluating the utility of non-invasive pre-transplant testing to detect coronary artery disease in terms of diagnostic yield and improved outcomes is conflicting. In the past, ESLD patients were believed to have a lower CAD risk than the general population due to lower cholesterol levels, blood pressure and peripheral vascular resistance. ${ }^{3}$ However, recent data have shown an increasing prevalence of $\mathrm{CAD}$ in this population which has been attributed to chronic inflammatory state, increased prevalence of diabetes mellitus, improving survival and higher smoking prevalence associated with some etiologies of ESLD., ${ }^{5}$ Despite the paucity of conclusive data supporting pre-transplant ischemic evaluation in asymptomatic patients, most societal guidelines currently recommend some type of physiologic testing such as stress echocardiography or

Funding None.

Reprint requests: Javier Gomez, MD, MSc, FACC, FASNC, Division of Cardiology, John H. Stroger Jr, Hospital of Cook County, 1901 W. Harrison St., Suite \# 3620, Chicago, IL 60612; jgomez@cookcountyhhs.org

J Nucl Cardiol 2021;28:2892-4.

$1071-3581 / \$ 34.00$

Copyright (c) 2020 American Society of Nuclear Cardiology. myocardial perfusion imaging (MPI) in these
population. ${ }_{7}$,

In this issue of the Journal, Moody et al. evaluated the incremental prognostic value of traditional cardiovascular risk factors, functional capacity and SPECTMPI on major cardiovascular adverse events in ESLD patients who were candidates for OLT. ${ }^{8}$ The authors evaluated 404 patients who were considered for OLT and had any of the following risk factors: inability to perform $>4$ METS by history, insulin-dependent diabetes mellitus, serum creatinine $>1.72 \mathrm{mg} / \mathrm{dL}$, history of CAD (defined as prior MI, PCI or CABG), stable angina, cerebrovascular disease or peripheral vascular disease. All patients underwent SPECT-MPI and those without known history of CAD underwent coronary artery calcium score (CACS). 244 patients were excluded from the primary analysis as they did not proceed with OLT and 2 patients were excluded due to early revascularization. The 158 remaining subjects were followed for a mean duration of 5.4 years for the outcomes of cardiac death, all-cause death and MACE (composite of cardiac death, non-fatal MI, malignant arrhythmia, ischemic stroke or hospitalization for congestive heart failure). The authors determined that abnormal perfusion as defined by SSS $\geq 4$ was prevalent in this high-risk population (20\%) and was associated with an increased risk of $\mathrm{CV}$ death with a hazard ratio of 4.2 (95\% CI 1.4-12.27 $p=0.019)$. Furthermore, subjects with abnormal SPECT-MPI and who were unable to exercise $\geq 4$ METS experienced the highest rate of $\mathrm{CV}$ and all cause death. The authors concluded that SPECT-MPI provides independent and incremental prognostic value over traditional cardiovascular risk factors and functional capacity in OLT candidates. The study findings suggest that it is reasonable to continue risk stratifying symptomatic and high risk asymptomatic OLT candidates with a non-invasive assessment that combines exercise capacity when feasible and SPECT-MPI. The authors should be commended for addressing a complex issue using a large 
cohort with long-term follow up. Some important points require further analysis.

A recent report by Duvall et al. summarized the 10year experience of a large teaching hospital and a transplant referral center in relation to the use of SPECT-MPI as part of the cardiovascular risk assessment of pre liver transplant patients. The study reported a low prevalence of abnormal SPECT-MPI $(7.3 \%)$ and even lower rate of obstructive CAD on invasive coronary angiography. These finding contrast those by Moody et al. in which the prevalence of abnormal MPI is significantly higher (20\%). The authors attribute this finding to a higher proportion or males, smokers and patients with diabetes mellitus in the cohort. Another contributing factor may be the impact of the stressing modality. Although exercise is generally the preferred stressing method to identify underlying myocardial ischemia, ESLD patients are frequently unable to achieve an adequate workload via exercise due to advanced illness. The underlying state of maximal vasodilation associated with ESLD has been considered, at least theoretically, a potential limitation to the ability of vasodilator stress to generate a significant vasodilatory response beyond the baseline state, raising concerns about its diagnostic and prognostic value. Moody et al. reported that approximately half of the patients (49\%) were able to complete the exercise stress protocol as compared to only $8.6 \%$ in the report by Duvall et al. No specific analysis evaluating the prognostic value of an abnormal SPECT-MPI after vasodilator stress is reported. Hence, it remains unclear whether vasodilator stress SPECT-MPI may account for the differences in prevalence of abnormal perfusion and whether it provides the same prognostic value as exercise stress SPECT-MPI for OLT candidates.

The parameters used by the authors to define high risk differ somewhat from the parameters used by the AHA/ACC consensus statement on evaluation and management among kidney and liver transplant candidates which defines high risk as the presence of $\geq 3$ of the following: diabetes mellitus, prior cardiovascular disease, left ventricular hypertrophy, age $>60$, smoking, hypertension and dyslipidemia., ${ }^{10}$ Although the parameters used by the authors have been established as cardiovascular risk factors, this difference in definitions limits the general applicability of the study results.

Coronary artery calcium score and cardiac CT angiography have emerged in the last decades as feasible and effective modalities to identify coronary artery disease in different subgroups. Previous reports have indicated that only CACS $\geq 400$ are associated with obstructive CAD as defined by $\geq 50 \%$ stenosis in $\geq 1$ coronary artery. ${ }^{11}$ Furthermore, OLT candidates with a CACS of zero have a very low probability of adverse
$\mathrm{CV}$ events. ${ }^{12}$ In the study cohort, most patients without known history of CAD had a CACS between zero and mild $(70 \%)$ and among those, the majority $(90 \%)$ had normal perfusion on SPECT-MPI. Moreover, although 4 subjects (10\%) with minimal CACS (1-10 Agatston units) were reported to have abnormal SPECT, only one patient had an adverse event over the 5 year follow up period. These findings suggest that CACS \pm CCTA, when feasible, may be a useful tool to exclude CAD in a large proportion of OLT candidates and more importantly, it may obviate the need for additional surveillance ischemic evaluation.

In conclusion, CAD appears to be more prevalent in OLT candidates than previously believed and pre-operative cardiac assessment has an important role in predicting long term outcomes. Symptomatic patients and high-risk asymptomatic patients stand to obtain the greatest benefit from this approach. Most reports on this topic are limited by small sample sizes, retrospective, single center design. It remains unclear whether revascularization in this patient population translates into improved outcomes. Future research focusing on multicenter, prospective data collection, perhaps creating a collaborative database between multiple large transplant centers focusing on the impact of the use of vasodilators as stress agents, outcomes after revascularization and the complementary value of emerging modalities such as CACS and CCTA, is needed to better guide future diagnostic and therapeutic interventions in this complex population.

\section{Disclosures}

No potential conflict of interest to declare.

\section{References}

1. Organ Procurement and Transplantation Network (2020) National Transplant Data by Organ

2. Parikh K, Appis A, Doukky R (2015) Cardiac imaging for the assessment of patients being evaluated for kidney or liver transplantation. J Nucl Cardiol 22:282-296

3. Ehtisham J, Altieri M, Salame E, Saloux E, Ollivier I, Hamon M (2010) Coronary artery disease in orthotopic liver transplantation: Pretransplant assessment and management. Liver Transpl 16:550557

4. Appleton CP, Hurst RT (2008) Reducing coronary artery disease events in liver transplant patients: Moving toward identifying the vulnerable patient. Liver Transpl 14:1691-1693

5. Poulin MF, Chan EY, Doukky R (2015) Coronary computed tomographic angiography in the evaluation of liver transplant candidates. Angiology 66:803-810

6. Lentine KL, Costa SP, Weir MR, Robb JF, Fleisher LA, Kasiske $\mathrm{BL}$ et al (2012) Cardiac disease evaluation and management among kidney and liver transplantation candidates: A scientific 
statement from the American Heart Association and the American College of Cardiology Foundation. J Am Coll Cardiol 60:434-480

7. Wolk MJ, Bailey SR, Doherty JU, Douglas PS, Hendel RC, Kramer CM et al (2014) ACCF/AHA/ASE/ASNC/HFSA/HRS/ SCAI/SCCT/SCMR/STS 2013 multimodality appropriate use criteria for the detection and risk assessment of stable ischemic heart disease: A report of the American College of Cardiology Foundation Appropriate Use Criteria Task Force, American Heart Association, American Society of Echocardiography, American Society of Nuclear Cardiology, Heart Failure Society of America, Heart Rhythm Society, Society for Cardiovascular Angiography and Interventions, Society of Cardiovascular Computed Tomography, Society for Cardiovascular Magnetic Resonance, and Society of Thoracic Surgeons. J Am Coll Cardiol 63:380-406

8. Moody WE, Holloway B, Arumugam P, Gill S, Wahid YS, Boivin $\mathrm{CM}$ et al (2020) Prognostic value of coronary risk factors, exercise capacity and single photon emission computed tomography in liver transplantation candidates: A 5-year follow-up study. J Nucl Cardiol. https://doi.org/10.1007/s12350-020-02126-Z
9. Duvall WL, Singhvi A, Tripathi N, Henzlova MJ (2018) SPECT myocardial perfusion imaging in liver transplantation candidates. $\mathrm{J}$ Nucl Cardiol 27:254

10. Alexander S, Teshome M, Patel H, Chan EY, Doukky R (2019) The diagnostic and prognostic utility of risk factors defined by the AHA/ACCF on the evaluation of cardiac disease in liver transplantation candidates. BMC Cardiovasc Disord 19:102

11. Kemmer N, Case J, Chandna S, Neff GW (2014) The role of coronary calcium score in the risk assessment of liver transplant candidates. Transplant Proc 46:230-233

12. Akincioglu C, Malhotra S (2018) Low yield of routine stress testing in patients awaiting liver transplantation. $\mathrm{J}$ Nucl Cardiol 27:266

Publisher's Note Springer Nature remains neutral with regard to jurisdictional claims in published maps and institutional affiliations. 